\title{
Genome-wide analysis validates aberrant methylation in fragile $X$ syndrome is specific to the FMR1 locus
}

Reid S Alisch ${ }^{2,5 \dagger}$, Tao Wang ${ }^{1,2 \dagger}$, Pankaj Chopra ${ }^{2}$, Jeannie Visootsak ${ }^{2,4}$, Karen N Conneely ${ }^{2}$ and Stephen T Warren ${ }^{2,3,4^{*}}$

\begin{abstract}
Background: Fragile $X$ syndrome (FXS) is a common form of inherited intellectual disability caused by an expansion of CGG repeats located in the $5^{\prime}$ untranslated region (UTR) of the FMR1 gene, which leads to hypermethylation and silencing of this locus. Although a dramatic increase in DNA methylation of the FMR1 full mutation allele is well documented, the extent to which these changes affect DNA methylation throughout the rest of the genome has gone unexplored.
\end{abstract}

Methods: Here we examined genome-wide methylation in both peripheral blood ( $\mathrm{N}=62)$ and induced pluripotent stem cells (iPSCs; $N=10$ ) from FXS individuals and controls.

Results: We not only found the expected significant DNA methylation differences in the FMR1 promoter and $5^{\prime}$ UTR, we also saw that these changes inverse in the FMR1 gene body. Importantly, we found no other differentially methylated loci throughout the remainder of the genome, indicating the aberrant methylation of FMR1 in FXS is locus-specific.

Conclusions: This study provides a comprehensive methylation profile of FXS and helps refine our understanding of the mechanisms behind FMR1 silencing.

Keywords: Epigenetics, DNA methylation, Fragile X syndrome

\section{Background}

Individuals with fragile X syndrome (FXS) exhibit a broad range of phenotypes, including varying degrees of intellectual disability, social impairment, macroorchidism, and an elongated face with large, everted ears. The most common mutation that causes FXS is an expansion of the CGG trinucleotide repeat located within the $5^{\prime}$ untranslated region (UTR) of the FMR1 gene [1-3]. When this expansion is greater than 200 repeats (known as the full mutation), the FMR1 promoter becomes hypermethylated, which prevents the expression of FMR1. Deletions and sequence variants within FMR1 result in a very small fraction of FXS

\footnotetext{
* Correspondence: swarren@emory.edu

${ }^{\dagger}$ Equal contributors

${ }^{2}$ Departments of Human Genetics, Emory University School of Medicine, 615

Michael Street, Atlanta, GA 30322, USA

${ }^{3}$ Departments of Biochemistry, Emory University School of Medicine, 615

Michael Street, Atlanta, GA 30322, USA

Full list of author information is available at the end of the article
}

cases [4-6], arguing that the loss of FMR1 function is the cause of FXS.

An association between the hypermethylation of the FMR1 trinucleotide repeats and FXS was first recognized over two decades ago [7], which sparked intensive study of the DNA methylation dynamics within the FMR1 locus. Research into the developmental timing of FMR1 silencing in chorionic villi $(\mathrm{CV})$ samples from FXS patients has revealed that the FXS full mutation alleles are still expressed during early embryogenesis (i.e. during gastrulation), indicating that epigenetic repression is established at a later developmental time point [8]. Furthermore, human embryonic stem cells (hESCs) derived from FXS patient embryos also express FMR1 in undifferentiated cells, until cellular differentiation triggers the recruitment of specific histone modifications, followed by DNA methylation and subsequent silencing of FMR1 transcription [9]. In contrast, the FMR1 locus remains hypermethylated in induced pluripotent stem (iPS) cell lines derived 
from FXS patients, suggesting that, once the methylation marks are established at this locus, they are stable and resistant to current reprograming methodologies [10].

A long-standing question in the field is whether the full mutation triggers DNA methylation elsewhere in the genome or only at the FMR1 locus. Resolving this question could modify theories of how an expanded CGG repeat triggers aberrant DNA hypermethylation. For example, RNA-induced transcriptional silencing (RITS) has been proposed as a mechanism to explain the silencing of FMR1 [11]. RITS is a form of gene silencing triggered by small interfering RNA (siRNA) and generally causes the transcriptional downregulation of a genomic region [12]. This model is attractive in that the unmethylated full mutation allele is known to be expressed in early development, presumably producing a transcript with a long riboCGG tract, and this riboCGG tract is cleaved in vitro by Dicer [13], producing small siRNA-like fragments of the riboCGG tract. Thus, it may be that small CGG RNAs could target chromatin-modifying activities back to the FMR1 locus. If true, there could be other CGG tracts in the genome that also are modified by this mechanism. To test this hypothesis, we examined DNA methylation levels at nearly half a million sites throughout the genome in the peripheral blood and fibroblast iPS cells of FXS patients using a highly sensitive genome-wide assay that quantitates methylation level at single $\mathrm{CpG}$ dinucleotide resolution; our results show that the hypermethylation of the FMR1 locus in FXS is indeed locus-specific.

\section{Methods}

\section{Human samples}

The study protocol and consent form used in this investigation wasreviewed and approved by the Emory Internal Review Board on August 3, 2012and given the approval number CR8_IRB00001764.

\section{Derivation of iPS cells}

Human normal fibroblasts CRL2097 were obtained from ATCC, and GM0011 (normal), GM05848, and GM07730 (fragile X patients) were obtained from the Coriell Cell Repositories. The fibroblasts were cultured in DMEM containing $10 \%$ FBS, $1 \times$ glutamine, $1 \times$ Non-Essential amino acids, and $1 \times$ Pen/Strep.

For human iPSC reprogramming, $1 \times 10^{5}$ fibroblasts were seeded in a well of a 6 -well plate. The next day, concentrated pMXs-hOCT4, hSOX2, hKLF4, and c-hMYC retrovirus were added to cells in the presence of 6 $\mu \mathrm{g} / \mathrm{ml}$ polybrene. A second round of transduction was repeated the following day. On day 7 after initial transduction, the cells were reseeded in $10-\mathrm{cm}$ dishes with irradiated MEF feeders. The hiPSC colonies were picked between days 18-25. iPSCs were maintained in hiPSC standard medium (DMEM/F12, 20\% KnockOut Serum Replacement,
1× MEM Non-Essential Amino Acids, $1 \times$ glutamine, 0.11 $\mathrm{mM}$ 2-mercaptoethanol, $10 \mathrm{ng} / \mathrm{ml} \mathrm{bFGF}$ ) on irradiated MEF feeders. The established iPSC cell lines were subsequently confirmed with AP staining, and pluripotent markers by immunofluorescence staining and the ability to differentiate into 3 germ layers. Before isolating genomic DNA, iPS cells were subcultured in mTeSR1 feeder free system (STEMCELL Technologies) for at least 3 passages to reduce potential contamination.

\section{DNA methylation profiling}

Five hundred nanograms of human genomic DNA was sodium bisulfite-treated for cytosine $(C)$ to thymine $(\mathrm{T})$ conversion using the EZ DNA Methylation-Gold kit (Zymo Research). The converted DNA was purified and prepped for analysis on the Illumina HumanMethylation450 BeadChips following the manufacturer's guidelines. Briefly, converted DNA was amplified, fragmented, and hybridized to the HumanMethylation450 pool of alleledifferentiating oligonucleotides. After a series of extension, ligation, and cleanup reactions, the DNA was labeled with a fluorescent dye. The labeled DNA was then scanned using an Illumina BeadArray Reader or iScan. Image analysis and signal determination were performed using the GenomeStudio software, Methylation Module (Illumina).

\section{Interpretation and QC of DNA methylation data}

CpG DNA methylation data were interpreted using GenomeStudio to quantify methylated (M) and unmethylated (U) signal intensities for genomic DNA. The signals were quantile normalized separately, and overall methylation levels $(\beta)$ were calculated as the ratio of methylated to total signal [i.e. $\beta=M /(M+U+100)$ ], where $\beta$ ranges from 0 (unmethylated) to 1 (methylated). Quality control of data resulted in removal of samples with aberrantly low signal intensity (mean <2000) or with fewer than 90\% of CpG loci detected, where a given locus was deemed not detected if the detection P-value was $>0.01$ (detection P-value provided by GenomeStudio and calculated relative to background signal). Any probe having more than 25\% detected P-values $>0.01$ was discarded from the analysis. Missing data were imputed using the "impute.knn" function from the "impute" package in R (Cran). Assay controls were inspected to remove samples with poor bisulfite conversion, staining, extension (single nucleotide extension assay), hybridization, or specificity. Furthermore, outliers identified by hierarchical clustering and/or dissimilarity matrices were removed. Additionally, one control DNA replicate was run on each BeadChip to assess overall assay reproducibility. Methylation profiles of the control DNA correlated well, with an average Pearson correlation coefficient (R) of 0.990 between replicates. 


\section{Analysis of FXS-associated $\mathrm{CpG}$ loci}

To analyze DNA methylation differences associated with FXS, we fit a separate regression for each CpG site. Although samples were randomly distributed across BeadChips and experiments with respect to disease, BeadChip was also included as a random effect covariate in all analyses to account for potential batch effects. The package "nlme" in R (Cran) was used for the mixed effect model. Fixed effects included in the model were intensity, position, and age (for blood). To test several FMR1-related and -unrelated hypotheses, we filtered the data to include only those probes that reside in the following genomic regions: 1500 base pairs of a transcription start site; 200 base pairs of a transcription start site; the first exon of a gene; the " 3 'UTR" of a gene; the " 5 'UTR" of a gene; a CpG Island; the "N_Shore" or "S_Shore" of a CpG island; or near a CGG trinucleotide repeat containing at least 8 consecutive repeats. We also filtered the data to include only those probes annotated to genes found to play a role in recurrent genomic abnormalities. To correct for multiple hypothesis testing, we applied a Benjamini-Hochberg False Discovery Rate (FDR) correction using the R function "p.adjust," but to avoid false positives due to the small sample size, we used conservative Bonferroni adjustment for our ultimate determination of significance.

\section{Permutation analyses}

All permutation analyses were conducted in $\mathrm{R}$ using the same linear model as the actual analysis, where BeadChip was treated as a mixed-effects covariate, but in each permutation the disease status of the subjects was randomly reassigned. In total, 1000 permutations were conducted for both the peripheral blood and iPS cell groups independently. Permutation P-values for each CpG locus were calculated by assessing the number of times each locus was more significantly associated with FXS in the 1000 permuted data sets than the actual association (Additional file 1: Figure S3; Additional file 2: Table S1 and Additional file 3: Table S2).

\section{Data access}

We have submitted the data generated from the 9 FXS samples and the 53 controls for this study to the Gene Expression Omnibus (GEO), which can be found under the Gene Series: GSE41273.

\section{Results}

We investigated FXS-associated ectopic DNA methylation changes using DNA extracted from the peripheral blood of nine FXS individuals and 53 healthy males. At the time of collection, these individuals ranged in age from $>1-48$ years (median 7.8; FXS; Additional file 4: Figure S1) and 3-18 years (median 10.3; controls; Additional file 4: Figure S1) and were epityped using Infinium HumanMethylation450
BeadChips, which provide a quantitative measure of DNA methylation denoted as $\beta$, calculated as the ratio of methylated to total DNA. This is a highly reproducible and widely used assay ([14-18] that measures $\beta$ at 485,512 CpG dinucleotides located proximal to the promoters of nearly all RefSeq genes. To determine whether FXS-associated DNA methylation changes were present in these individuals, we analyzed each locus using a linear mixed-effects regression model that adjusted for age (see Methods) and identified 17 differentially methylated probes, 15 of which are annotated to the FMR1 promoter or gene body: 14 FXS-methylated loci and 1 FXS-demethylated locus (Bonferroni <0.05; Figure $1 \mathrm{~A}, \mathrm{~B}$; Additional file 2: Table S1 and Additional file 3: Table S2). Though the 15 FMR1-associated loci exhibit a unique FXS-associated DNA methylation profile of distinct methylation levels with little overlap for FXS samples versus controls (Figure 1B), the two autosomal differentially methylated loci (KLK15 and MICA) were not so distinct; they were also hypomethylated rather than hypermethylated and were not flanked by other significantly differentially methylated loci, as one sees at FMR1 (Additional file 5: Figure S2). This suggests these two autosomal loci are not modified similar to FMR1 in full mutation individuals, and the results for these two loci may be false positives due to our small sample size or possible population substructure of the epigenome. To validate our methods statistically and adjust for possible failure of asymptotic assumptions due to the small sample size, we performed a thousand permutations of the data (see Methods). For the 15 FMR1-associated probes, the P-values from the original analysis were always lower than the $1000 \mathrm{P}$-values obtained in the permuted datasets, suggesting an empirical $\mathrm{P}$-value of $<.001$ (Additional file 1: Figure S3; Additional file 2: Table S1; Methods). Since differential methylation of the FMR1 allele is well established [7] and in direct agreement with our findings, these differences were not validated using an alternate molecular assay.

We next tested several FXS-related and -unrelated hypotheses, including recurrent genomic abnormalities associated with intellectual delay and annotated genomic structures (e.g. CpG islands; Methods). Although this approach reduces our multiple testing burden and effectively increases our power to find subtle yet significant changes in DNA methylation, as before the only probes identified as differentially methylated were probes annotated to the FMR1 gene (data not shown). Note that even for the strict Bonferroni criteria employed for the genome-wide analysis, $75 \%$ of the CpG sites had $>80 \%$ power to detect $\beta$ - value differences of 0.10 or greater, so the lack of observed widespread methylation differences in genes other than FMR1 cannot be explained by low power. Finally, to gain insight into the potential mechanism(s) behind FXS-associated DNA methylation changes, we 


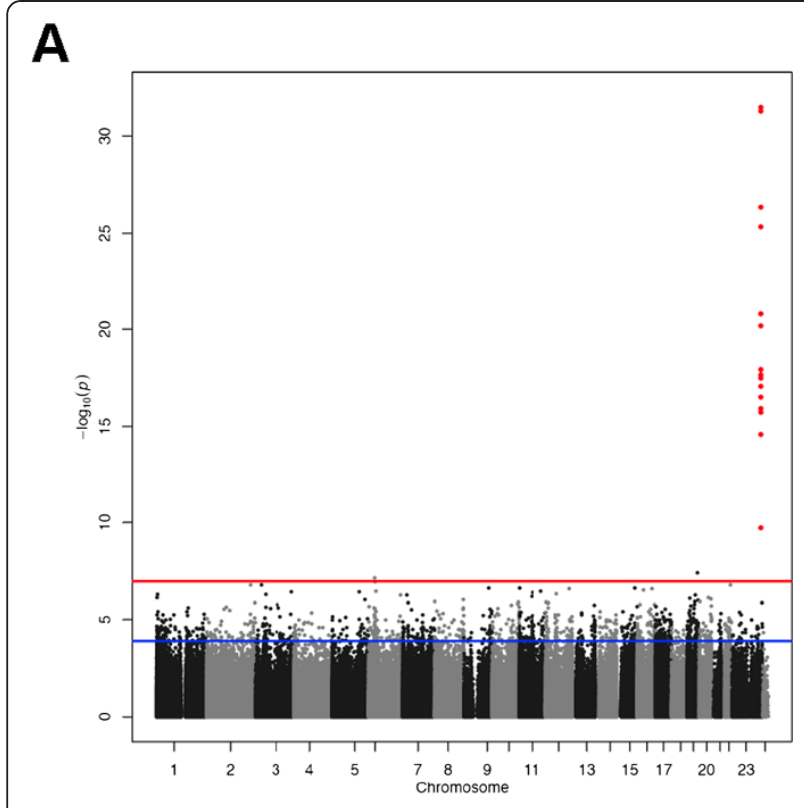

C

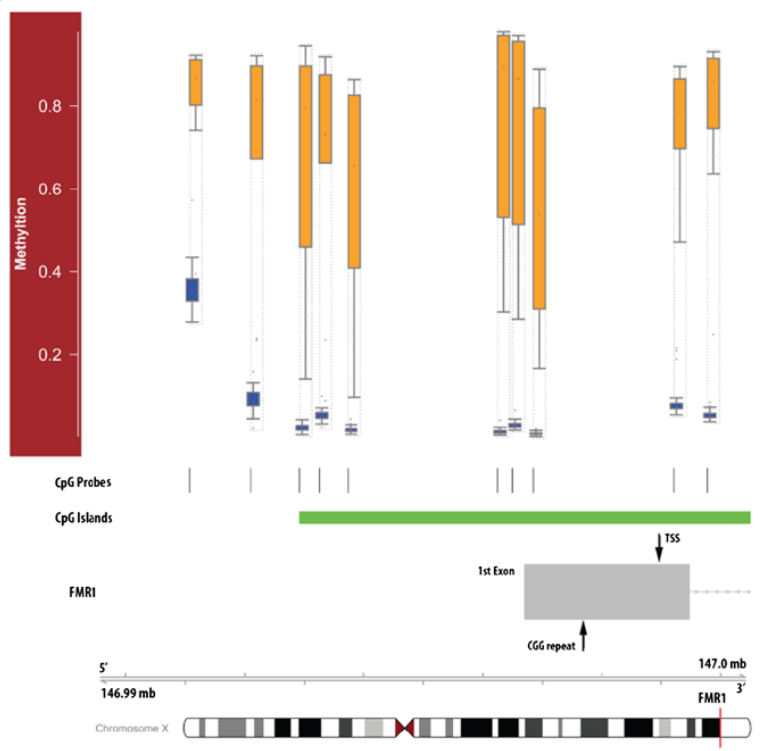

B

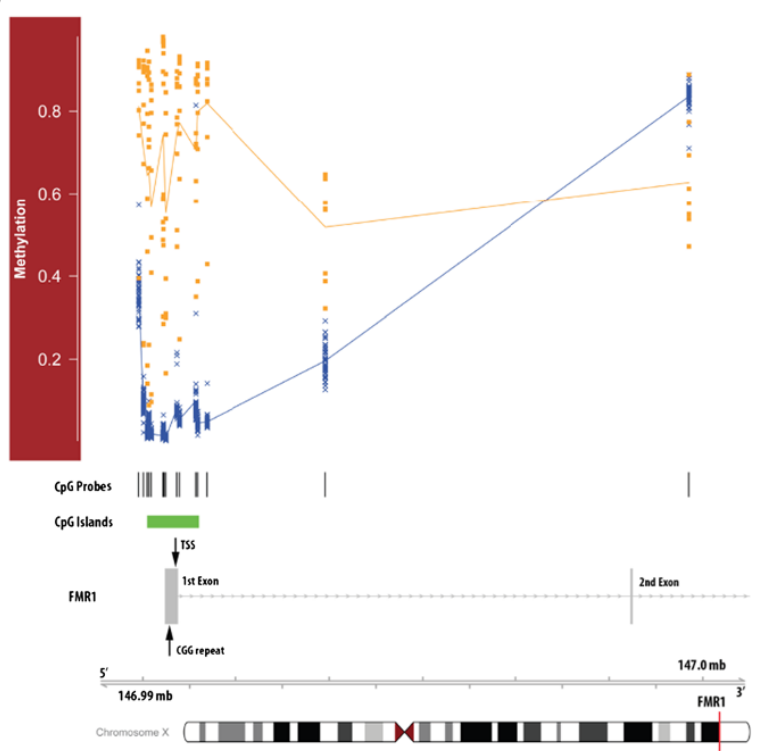

D

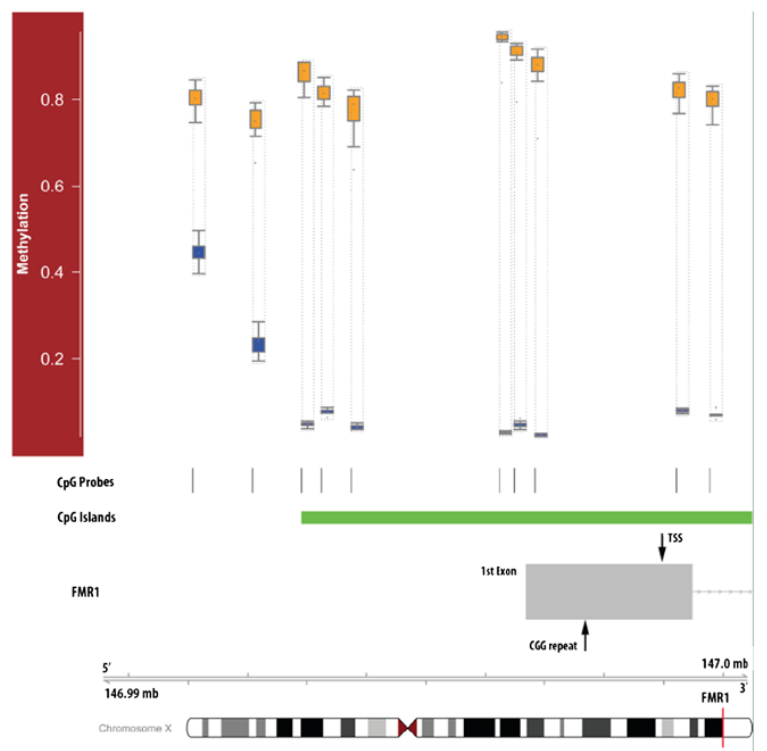

Figure 1 FXS-associated differentially methylated loci. (A) Modified Manhattan plot of FXS-associated methylation levels in the peripheral blood: loci are displayed with the -log10(P-value) generated by the linear mixed-effect model ( $y$-axis). Horizontal lines reflect cutoffs for FDR $<0.05$ (blue line) and Bonferroni-adjusted P-value $<0.05$ (red line). FMR1 annotated loci are shown in red; otherwise, loci are colored black or gray on alternating chromosomes (x-axis). (B) Relative locations of FMR1 methylation. Top panel shows the methylation levels (y-axis) in FXS (orange squares) and control (blue crosses) individuals at several loci annotated in or near the FMR1 locus. Shown below are the relative CpG coverage (vertical black lines) and CpG island location (green rectangle). Bottom panel depicts a gene schematic of FMR1, indicating the relative location of the CGG repeat (expanded in FXS), the FMR1 transcription start site (TSS), and exons 1 and 2. The ideogram of the X chromosome (bottom) shows the relative location of FMR1 (red vertical bar), and the genomic position shown above is relative to HG-19 coordinates. (C and D) FMR1 promoter DNA methylation levels in peripheral blood and iPS cells. Top panel shows box and whisker plots of the methylation levels ( $y$-axis) in FXS (orange) and control (blue) individuals at several loci annotated in the FMR1 promoter. Bottom panel is described in B. 
annotated the genome for all CGG trinucleotide repeats containing at least eight consecutive repeats $(\mathrm{N}=136$ tracts; $\mathrm{N}=452$ probes; see Methods) and found two juxtaposed probes annotated to $Z F H X 3$ that reached significance (Additional file 6: Table S3). In contrast to the FMR1 locus, the ZFHX3 locus had no distinct FXS-associated DNA methylation or gene expression differences (Additional file 7: Figure S4; data not shown). These findings imply that the significant DNA methylation differences observed at the two probes do not have a functional consequence on $Z F H X 3$ expression. Together, these data suggest that the FXS-associated hypermethylation of the FMR1 promoter is locus-specific and does not alter DNA methylation elsewhere in the genome.

To corroborate these findings, we derived a total of ten iPS cell lines from fibroblasts of two FXS patients (FXS-iPS) and two control individuals (iPS). DNA was extracted from 12 FXS-iPS cell lines and 11 iPS cell lines and epityped using Infinium HumanMethylation450 BeadChips. Limiting the FXS-associated DNA methylation analysis in this group to only those loci that satisfied an FDR $<0.05$ in the peripheral blood analysis $(\mathrm{N}=1183$ probes; Figure 1A; see Methods) yielded results similar to those found in peripheral blood: eight FXS-methylated loci and one FXS-demethylated locus; all nine differentially methylated loci are annotated to the FMR1 promoter (Bonferroni <0.05) (Figure 1C; Additional file 8: Table S4). Since iPS cells show significant reprogramming variability, we also excluded the hotspots of aberrant reprogramming regions reported by Lister et al. [19] and still found FXS-associated DNA methylation changes only at the FMR1 locus (data not shown). Subsequent hypothesis- and mechanism-driven analyses also failed to uncover any non-FMR1 annotated FXS-associated DNA methylation changes, including at the ZFHX3 locus. Therefore, FXS-derived iPS cells exhibit similar genome-wide methylation profiles as terminally differentiated cells of blood, a FMR1-specific epigenetic disruption.

\section{Discussion}

This study provides a sensitive and comprehensive quantitative analysis of genome-wide DNA methylation levels in a group of FXS and control individuals. We found that FXSassociated hypermethylation is profound throughout the CpG island encompassing the FMR1 $5^{\prime}$ UTR, revealing CpG dinucleotides whose distinct FXS methylation profile could improve current diagnostic methods. For example, there are four CpG loci in FMR1 that show a clear distinction between all FXS and control samples with no overlap (Figure 1B), suggesting that interrogation of these loci for methylation would be diagnostic. The finding that FXSassociated methylation is significantly decreased at one CpG in the FMR1 gene body is consistent with previous reports indicating that gene body hypermethylation is associated with active gene expression [20]. It would be interesting to explore whether this trend persists throughout the remainder of the gene.

Our examination reported here shows that CGG repeats elsewhere in the genome do not appear abnormally methylated in trans with the full mutation. Thus, either the hypothesis of a RITS role in silencing of FMR1 is false, or there may be a threshold of length for a CGG repeat tract to be susceptible to silencing, since there are no known CGG tracts in the reference genome that even approach the size of a premutation, let alone a full mutation allele. Indeed, the expression of the normal FMR1 allele opposite the full mutation in FXS females would be consistent with a threshold model.

\section{Conclusion}

When FMR1 was first identified, the question posed in this study was unanswerable. Today, our knowledge of the human genome sequence allows genome-wide examination of DNA methylation differences. Here we report that only probes located in the FMR1 promoter or gene body exhibit FXS-associated DNA methylation differences in DNA from peripheral blood and iPS cells of FXS individuals. Thus, while this study does not determine the mechanism behind the aberrant methylation in the expanded FMR1 repeat, it does help refine our mechanistic picture of FMR1 silencing in fragile $\mathrm{X}$ syndrome. Since we did not find any nonFMR1-associated differentially methylated loci, we have made a significant stride toward finally put a long-standing question in FXS research to rest.

\section{Additional files}

Additional file 1: Figure S3. Permutation analysis of FXS-associated loci. Scatterplot of permuted (1000 permutations) FXS-associated $P$-values ( $x$-axis) compared to asymptotic $P$-values (y-axis) calculated using the linear model (Pearson $\mathrm{R}=0.997$ ).

Additional file 2: Table S1. Summary of the linear mixed-effects regression model. Column headers include: Illumina identification name (cpgids); Bonferroni-corrected P-values (bonferroni); False discovery rate P-values (fdr); Uncorrected P-value (raw_pvalue); Permutation P-value (perm_pvalue); Chromosome location (CHR); HG19 Map position (MAPINFO); and RefSeq gene name (UCSC_RefGene_Name).

Additional file 3: Table S2. Summary of the mean differences in methylation between FXS and control individuals. Column headers include: Illumina identification name (cpgids); Mean raw beta-values for fragile X patients (Mean FXS); Mean raw beta-values for control individuals (Mean controls); Chromosome location (CHR); HG19 Map position (MAPINFO); and RefSeq gene name (UCSC_RefGene_Name).

Additional file 4: Figure S1. Sample age distribution. The frequency (yaxis) of FXS (orange) and control (blue) individuals at each age (x-axis), with mean ages denoted by the vertical dashed lines.

Additional file 5: Figure S2. Methylation levels and significance of non-FMR1 FXS-associated loci. Top two panels show the methylation levels (y-axis) in FXS (orange squares) and control (blue crosses) individuals at several loci near the two significant non-FMR1 FXSassociated loci. Bottom two panels depict the P-values (-log(P-value); yaxis) for each probe generated by the mixed-effect linear model. Red line 
indicates the Bonferroni cutoff of 0.05 . Only one probe from each region shown is significant at $P<0.05$. Position of probes ( $x$-axis) in all four panels is relative to HG-19 coordinates for chromosomes 19 and 6, KLK15 and MICA, respectively.

Additional file 6: Table S3. Summary of the linear mixed-effects regression model using the data set filtered for all CGG trinucleotide repeats containing at least eight consecutive repeats ( $N=136$ tracts; $N=$ 452 probes). Column headers include: Illumina identification name (cpgids); Bonferroni-corrected P-values (bonferroni); False discovery rate P-values (fdr); Uncorrected P-value (raw_pvalue); Permutation P-value (perm_pvalue); Chromosome location (CHR); HG19 Map position (MAPINFO); and RefSeq gene name (UCSC_RefGene_Name).

Additional file 7: Figure S4. Methylation levels of ZFHX3. All panels show the methylation levels ( $y$-axis) in FXS (orange squares) and control (blue crosses) individuals at loci annotated to ZFHX3. Top panel (All CpG probes) shows the methylation levels of all the probes annotated to ZFHX3. Middle panel (Probes in a CGG repeat) displays only those ZFHX3 probes (4) that reside in a CGG trinucleotide repeat containing at least eight consecutive repeats. The bottom panel (Significant CGG repeat probes) shows the two ZFHX3-annotated probes that are significantly different (Bonferroni $<0.05$ ) between FXS and control individuals. Position of probes (x-axis) in all three panels is relative to HG-19 coordinates for chromosome 16

Additional file 8: Table S4. Summary of the linear mixed-effects regression model using the IPS cell data set filtered loci that satisfied an FDR $<0.05$ in the peripheral blood analysis ( $N=1183$ probes). Column headers include: Illumina identification name (cpgids); Bonferronicorrected P-values (bonferroni); False discovery rate P-values (fdr): Uncorrected P-value (raw_pvalue); Chromosome location (CHR); HG19 Map position (MAPINFO); and RefSeq gene name (UCSC_RefGene_Name).

\section{Abbreviations}

FXS: Fragile X syndrome; UTR: Untranslated region; CV: Chorionic villi; hESCs: Human embryonic stem cells; iPS: Induced pluripotent stem; C: Cytosine; T: Thymine; M: Methylated; U: Unmethylated; $\beta$ : Methylation levels; R: Pearson correlation coefficient; RITS: RNA-induced transcriptional silencing; siRNA: Small interfering RNA.

\section{Competing interests}

The authors declare that they have no competing interests.

\section{Authors' contribution}

RSA designed experiments, generated and interpreted all methylation data and wrote manuscript. TW designed experiments, generated expression data and wrote manuscript. PC designed and analyzed all methylation data. JV designed recruitment protocol and collected samples. KNC developed analytical methods of methylation data. STW designed experiments, interpreted data and wrote manuscript. All authors read and approved the final manuscript.

\section{Acknowledgements}

The authors would like to thank Julie Mowrey and Brian Lynch for technical assistance and Cheryl Strauss for editorial comments. This work was supported in part by a Simons Foundation (SFARI) award and $\mathrm{NIH}$ grants MH089606 and HD24064, all to STW. We are grateful to all the families collected from the Department's Fragile X Syndrome Clinic and those participating at the SFARI Simplex Collection (SSC) sites, as well as the principal investigators (A. Beaudet, R. Bernier, J. Constantino, E. Cook, E. Fombonne, D. Geschwind, D. Grice, A. Klin, D. Ledbetter, C. Lord, C. Martin, D. Martin, R. Maxim, J. Miles, O. Ousley, B. Peterson, J. Piggot, C. Saulnier, M. State, W. Stone, J. Sutcliffe, C. Walsh, and E. Wijsman). We also appreciate the access to phenotypic data on SFARI Base. Approved researchers can obtain the SSC population dataset described in this study by applying at https:// base.sfari.org. Emory University's research IT service center and its high performance computer cluster also supported this research.

\section{Author details}

${ }^{1}$ Genetics and Molecular Biology Graduate Program, Emory University, Atlanta, GA 30322, USA. '2Departments of Human Genetics, Emory University
School of Medicine, 615 Michael Street, Atlanta, GA 30322, USA.

${ }^{3}$ Departments of Biochemistry, Emory University School of Medicine, 615 Michael Street, Atlanta, GA 30322, USA. ${ }^{4}$ Departments of Pediatrics, Emory University School of Medicine, 615 Michael Street, Atlanta, GA 30322, USA.

${ }^{5}$ Current address: Department of Psychiatry, University of Wisconsin-Madison, Madison, WI 53719, USA.

Received: 23 October 2012 Accepted: 25 January 2013

Published: 29 January 2013

\section{References}

1. Verkerk AJ, Pieretti M, Sutcliffe JS, Fu YH, Kuhl DP, Pizzuti A, Reiner O, Richards S, Victoria MF, Zhang FP, et al: Identification of a gene (FMR-1) containing a CGG repeat coincident with a breakpoint cluster region exhibiting length variation in fragile X syndrome. Cell 1991, 65(5):905-914

2. Wang T, Bray SM, Warren ST: New perspectives on the biology of fragile $X$ syndrome. Curr Opin Genet Dev 2012, 22(3):256-263.

3. Ashley CT, Sutcliffe JS, Kunst CB, Leiner HA, Eichler EE, Nelson DL, Warren ST: Human and murine FMR-1: alternative splicing and translational initiation downstream of the CGG-repeat. Nat Genet 1993, 4(3):244-251

4. Coffee B, Ikeda M, Budimirovic DB, Hjelm LN, Kaufmann WE, Warren ST Mosaic FMR1 deletion causes fragile $\mathrm{X}$ syndrome and can lead to molecular misdiagnosis: a case report and review of the literature. Am J Med Genet A 2008, 146A(10):1358-1367.

5. De Boulle K, Verkerk AJ, Reyniers E, Vits L, Hendrickx J, Van Roy B, Van den Bos F, de Graaff E, Oostra BA, Willems PJ: A point mutation in the FMR-1 gene associated with fragile X mental retardation. Nat Genet 1993, 3(1):31-35.

6. Lugenbeel KA, Peier AM, Carson NL, Chudley AE, Nelson DL: Intragenic loss of function mutations demonstrate the primary role of FMR1 in fragile $X$ syndrome. Nat Genet 1995, 10(4):483-485.

7. Bell MV, Hirst MC, Nakahori Y, MacKinnon RN, Roche A, Flint TJ, Jacobs PA, Tommerup N, Tranebjaerg L, Froster-Iskenius U, et al: Physical mapping across the fragile $X$ : hypermethylation and clinical expression of the fragile X syndrome. Cell 1991, 64(4):861-866.

8. Willemsen R, Bontekoe CJ, Severijnen LA, Oostra BA: Timing of the absence of FMR1 expression in full mutation chorionic villi. Hum Genet 2002, 110(6):601-605.

9. Eiges R, Urbach A, Malcov M, Frumkin T, Schwartz T, Amit A, Yaron Y, Eden A, Yanuka O, Benvenisty $N$, et al: Developmental study of fragile $X$ syndrome using human embryonic stem cells derived from preimplantation genetically diagnosed embryos. Cell Stem Cell 2007, 1(5):568-577.

10. Urbach A, Bar-Nur O, Daley GQ, Benvenisty N: Differential modeling of fragile $X$ syndrome by human embryonic stem cells and induced pluripotent stem cells. Cell Stem Cell 2010, 6(5):407-411.

11. Jin $P$, Alisch RS, Warren ST: RNA and microRNAs in fragile $X$ mental retardation. Nat Cell Biol 2004, 6(11):1048-1053.

12. Verdel A, Jia S, Gerber S, Sugiyama T, Gygi S, Grewal SI, Moazed D: RNAi-mediated targeting of heterochromatin by the RITS complex. Science 2004, 303(5658):672-676.

13. Handa V, Saha T, Usdin K: The fragile $X$ syndrome repeats form RNA hairpins that do not activate the interferon-inducible protein kinase, PKR, but are cut by Dicer. Nucleic Acids Res 2003, 31(21):6243-6248.

14. Bork S, Pfister S, Witt H, Horn P, Korn B, Ho AD, Wagner W: DNA methylation pattern changes upon long-term culture and aging of human mesenchymal stromal cells. Aging Cell 2010, 9(1):54-63.

15. Teschendorff AE, Menon U, Gentry-Maharaj A, Ramus SJ, Weisenberger DJ, Shen H, Campan M, Noushmehr H, Bell CG, Maxwell AP, et al: Agedependent DNA methylation of genes that are suppressed in stem cells is a hallmark of cancer. Genome Res 2010, 20(4):440-446.

16. Chen YA, Choufani S, Ferreira JC, Grafodatskaya D, Butcher DT, Weksberg R Sequence overlap between autosomal and sex-linked probes on the Illumina HumanMethylation27 microarray. Genomics 2011, 97(4):214-222.

17. Koch CM, Suschek CV, Lin Q Bork S, Goergens M, Joussen S, Pallua N, Ho $A D$, Zenke $M$, Wagner W: Specific age-associated DNA methylation changes in human dermal fibroblasts. PLoS One 2011, 6(2):e16679.

18. Alisch RS, Barwick BG, Chopra P, Myrick LK, Satten GA, Conneely KN, Warren ST: Age-associated DNA methylation in pediatric populations. Genome Res 2012, 22(4):623-632. 
19. Lister R, Pelizzola M, Kida YS, Hawkins RD, Nery JR, Hon G, AntosiewiczBourget J, O'Malley R, Castanon R, Klugman S, et al: Hotspots of aberrant epigenomic reprogramming in human induced pluripotent stem cells. Nature 2011, 471(7336):68-73.

20. Hellman A, Chess A: Gene body-specific methylation on the active $X$ chromosome. Science 2007, 315(5815):1141-1143.

doi:10.1186/1471-2350-14-18

Cite this article as: Alisch et al:: Genome-wide analysis validates aberrant methylation in fragile $\mathrm{X}$ syndrome is specific to the FMR1 locus. BMC Medical Genetics 2013 14:18.

\section{Submit your next manuscript to BioMed Central} and take full advantage of:

- Convenient online submission

- Thorough peer review

- No space constraints or color figure charges

- Immediate publication on acceptance

- Inclusion in PubMed, CAS, Scopus and Google Scholar

- Research which is freely available for redistribution 